\title{
Stickoxid in Exspirationsluft: Entzündungsparameter bei Asthmatikern?
}

\author{
Die Entdeckungsgeschichte rund ums Stickoxid (NO) reißt nicht \\ ab. Nachdem die Rolle des kleinen anorganischen Moleküls bei \\ der Arteriosklerose längst gesichert ist, verdichten sich nun die \\ Hinweise, daß NO auch bei immunologischen Prozessen beteiligt \\ ist. Eine Londoner Arbeitsgruppe kommt nach einer experimentel- \\ len Studie zu dem Schluß, daß der erhöhte NO-Anteil in der \\ Exspirationsluft von Asthmatikern auf die inflammatorischen \\ Vorgänge im unteren Respirationstrakt zurückgeht - womit sich \\ NO potentiell als Marker für die Krankheitsaktivität eignen würde.
}

tickoxide (NO) werden von einer Vielzahl der Zellen des Respirationstraktes gebildet. Sie scheinen eine wichtige Rolle bei der physiologischen Funktion der Atemwege als auch bei der Pathophysiologie der Atemwegserkrankungen zu spielen.

Schon seit einigen Jahren ist bekannt, daß Asthmatiker eine erhöhte NO-Konzentration in der Exspirationsluft aufweisen. Möglicherweise ist dies Folge einer Stimulation der NO-Synthase durch inflammatorische Zytokine, endgültig geklärt ist dieser Zusammenhang bisher aber nicht. Eine Londoner Arbeitsgruppe hat daher jetzt in einer experimentellen Studie zu klären versucht, woher das vermehrte NO bei Asthmatikern stammt.

\section{Stickoxidmessung bei verschiedenen Atem- manövern}

Mittels Chemilumineszenz-Analyse wurde der NO-Gehalt in der Ausatemluft von 30 Asthmatikern und 46 gesunden Personen unter verschiedenen
Atemmanövern gemessen. Bei Exspiration ohne Widerstand war der NOGehalt bei den Asthmatikern signifikant höher als bei den Gesunden (46 \pm 6 ppb vs. $7 \pm 1$ ppb, $\mathrm{p}<0,001)$. In noch größerem Ausmaß galt dies für die Exspiration gegen Widerstand (301 \pm 26 ppb vs. $78 \pm 3$ ppb, $\mathrm{p}<0,001)$ (s. Abb.). Das Atemanhalten für 20 Sekunden resultierte in einem initialen NO-Peak (Asthmatiker: 172 \pm 29 ; Gesunde: $13 \pm 1, \mathrm{p}<0,001)$ mit end-ex- spiratorischen NO-Werten wie bei der Exspiration ohne Widerstand.

Eine bronchoskopische Probenentnahme aus Trachea und Mittellappenbronchus bei 15 Asthmapatienten und 5 gesunden Probanden zeigte ähnliche NO-Werte wie bei der Exspiration ohne Widerstand. Die NO-Konzentration in der Nase lag bedeutend über der in der Exspirationsluft und war ebenfalls bei Asthmatikern signifikant erhöht $(1390 \pm 71$ ppb vs. $996 \pm 39$ ppb, $\mathrm{p}<0,002)$. Allerdings korrelierten die nasalen NO-Werte nicht mit den exspiratorischen NO-Werten.

\section{Erhöhtes NO bei Asthmapatienten aus unterem Respirationstrakt}

Die Autoren kommen zu folgenden Schlußfolgerungen: Da der Unterschied in den NO-Werten zwischen Asthmatikern und Gesunden am ausgeprägtesten in der Exspiration ohne Widerstand war und zugleich bekannt ist, daß sich bei der Exspiration gegen Widerstand nasale NO-Anteile in die Exspirationsluft mengen, sind die erhöhten NO-Werte bei Asthmapatienten offenbar auf den unteren Respirationstrakt zurückzuführen. Hierfür spricht auch der erhöhte NO-Wert in den Gewebeproben aus Trachea und Bronchus bei Asthmatikern.

Der wahrscheinlichste Ursprung der erhöhten NOWerte bei Asthmapatienten sind die Alveolar- sowie die Endothelzellen der Luftwege. Offenbar induzieren hier die bei Asthma vermehrten proinflammatorischen Zytokine die NO-Synthase-Produktion. Ein weiteres Indiz hierfür ist, daß unter inhalativer Kortikoidtherapie der NO-Gehalt in der Exspirationsluft deutlich sinkt.

Zusammenfassend könnte sich der NO-Gehalt in der Ausatemluft nach Ansicht der Autoren damit als Marker für die pulmonale Entzündungsaktivität bei Asthmatikern eignen.

$(z o)$

Stickoxidwerte in der Exspirationsluft von 30 Asthmapatienten und 46 gesunden Probanden. Die Exhalition wurde gegen Widerstand durchgeführt, eingezeichnet sind jeweils die Spitzenwerte. Die waagerechte Linie kennzeichnet den jeweiligen Mittelwert.
Quelle: Kharitonov SA, Chung KF, Evans D, nitric oxide in asthma is mainly derived from the lower respiratory tract. Am J Respir Crit Care Med 1996; 153: 1773-1780. O'Connor BJ, Barnes PJ. Increased exhaled 